\title{
Publisher Correction: Global fire emissions buffered by the production of pyrogenic carbon
}

Matthew W. Jones (D, Cristina Santín (D), Guido R. van der Werf and Stefan H. Doerr

Correction to: Nature Geoscience https://doi.org/10.1038/s41561-019-0403-x, published online 5 August 2019.

In the version of this Article originally published, the supplementary data file containing the global dataset of the PyC production factors was not uploaded with the paper.

This file has now been uploaded as Supplementary Data 1, and the first sentence of the Data availability statement was updated from: 'The global dataset of the PyC production factors is available as a supplementary data file (GlobalPyC_supplementarydataset.xlsx).' to 'The global dataset of the PyC production factors is available in Supplementary Data 1.'

This has now been corrected.

Published online: 1 June 2020

https://doi.org/10.1038/s41561-020-0600-7

(C) The Author(s), under exclusive licence to Springer Nature Limited 2020 